\title{
EVALUACIÓN DE SIETE POBLACIONES DE ESPÁRRAGO (ASPARAGUS OFFICINALIS L.) ${ }^{1}$
}

\author{
ILEANA GATTI ${ }^{2}$, VANINA PAMELA CRAVERO ${ }^{3}$, FERNANDO SEBASTIÁN LÓPEZ ANIDO ${ }^{4}$ y ENRIQUE LUIS COINTRY 5
}

\begin{abstract}
RESUMEN - Los materiales de espárrago (Asparagus officinalis L.) utilizados hasta el momento por los productores son introducciones realizadas por las casas semilleras y han sido seleccionados para satisfacer requerimientos del mercado de los sitios de origen. El objetivo del siguiente trabajo es evaluar siete poblaciones de espárrago $\left(\mathrm{P}_{1}\right.$ a $\left.\mathrm{P}_{7}\right)$, con el fin de seleccionar genitores adecuados de manera de recurrir a la hibridación de ellos para obtener materiales adaptados a los requerimientos locales. Las evaluaciones se hicieron sobre plantas individuales, separadas por sexos y manejadas como espárrago blanco, durante los años 1993 y 1994, en el campo experimental de la Facultad de Ciencias Agrarias (Universidad Nacional de Rosario), ubicado en Zavalla, provincia de Santa Fe. La evaluación se realizó sobre planta individual, durante un período de 40 días de cosecha y con los datos obtenidos se realizó un ANOVA y un análisis de agrupamiento. Para elegir progenitores femeninos con altos rendimientos y rendimiento de mercado se determinó que se deberá recurrir a las $\mathrm{P}_{1}, \mathrm{P}_{2}$ y $\mathrm{P}_{3}$, las cuales presentan también alto número de turiones. Para altos peso medio y diámetro de turión, así como producción tardía, son indicadas las $\mathrm{P}_{5}$ y $\mathrm{P}_{7}$ como genitores masculinos, teniendo en cuenta que, mientras la $\mathrm{P}_{7}$ presenta bajo rendimiento, la $\mathrm{P}_{5}$ aportaría mejores producciones.
\end{abstract}

Términos para indexación: mejoramiento vegetal, caracterización de poblaciones, análisis de agrupamientos.

\section{EVALUATION OF SEVENASPARAGUS POPULATIONS (ASPARAGUSOFFICINALISL.)}

ABSTRACT - The materials of asparagus (Asparagus officinalis L.) that growers have in use are imported by seed dealers and have been selected according to overseas markets requirements. With the aim of selecting proper parents to use in a breeding program, seven populations of Asparagus officinalis $\left(\mathrm{P}_{1}\right.$ to $\left.\mathrm{P}_{7}\right)$, all rised as white asparagus, were tested during 1993 and 1994, at the Experimental Field of the Facultad de Ciencias Agrarias (Universidad Nacional de Rosario) placed in Zavalla, Santa Fe, Argentina. The evaluation was made on individual plant in a 40 days of harvest period. ANOVA and a cluster analysis were made. In order to improve total and marketable yield, $\mathrm{P}_{1}, \mathrm{P}_{2}$ and $\mathrm{P}_{3}$ should be used as females regarding that these ones also have high spear number. To improve spears weight and diameter as well as late production, $\mathrm{P}_{5}$ and $\mathrm{P}_{7}$ are recommended as males, but it should be considered that $\mathrm{P}_{5}$ has a better yield performance.

Index terms: plant breeding, population characterization, cluster analysis.

\section{INTRODUCCIÓN}

El espárrago es una planta dioica que se reproduce fundamentalmente por semilla. Su cultivo es

${ }^{1}$ Aceptado para publicación en 25 de mayo de 1999.

${ }^{2}$ Ing. Agr., Becaria del CONICET, Cátedra de Genética, Fac de Cs. Agrarias, Universidad Nacional de Rosario (UNR), CC 14 Zavalla 2123, Santa Fe, Argentina. E-mail: alegro@arnet.com.ar

${ }^{3}$ Lic. en Genética, Ayud. 1a Fac. Cs. Agr., UNR.

${ }^{4}$ Ing. Agr., M.Sc., J.T.P., Fac. Cs. Agr., UNR.

${ }^{5}$ Ing. Agr., M.Sc., Prof. Adjunto, Fac. Cs. Agr., UNR. plurianual, con elevados costos de producción debidos fundamentalmente a la mano de obra requerida durante la cosecha. Debido a estos altos costos el resultado económico del cultivo en la zona es bajo y para mejorarlo sería necesario contar con materiales de adaptación local, de altos rendimientos, que mantengan buenas características de calidad y que a su vez entren en producción más tardíamente (noviembre/diciembre), con el objetivo de exportar al hemisferio norte en contraestación.

Debido a la característica plurianual del cultivo, el período de evaluación es prolongado, pero, según 
Corriols (1983) y Fallon \& Nikoloff (1986) la evaluación del rendimiento durante los dos primeros años de cosecha se correlaciona altamente con la productividad en períodos más prolongados, y por lo tanto, puede utilizarse como estimador del desempeño de los materiales. Otros autores afirman que, al ser el espárrago una planta dioica de polinización cruzada, presenta diferencias sexuales en parámetros productivos y de calidad de turión. Ellison et al. (1960), Moon (1976), Fallon \& Nikoloff (1986) y Gonzales Castañón (1990) afirman que las plantas estaminadas presentan mayor rendimiento mientras que las pistiladas presentan mayor diámetro de turión. En algunas poblaciones no existe esta diferencia en rendimiento por compensación entre el número de turiones y su diámetro (Thevenin, 1967; Bannerot et al., 1969; Ley et al., 1976; Cointry et al., 1996b). La existencia o no de dichas diferencias permite conformar grupos homogéneos y identificar a aquellos materiales más aptos para actuar como genitores masculinos o femeninos, según las características que aporten, en la construcción de materiales híbridos, ya sean dobles, tres vías, clonales o todo macho (TM), los cuales presentan importantes progresos en la productividad con respecto a los materiales de partida, como fue ya señalado por algunos autores (Thevenin, 1967; Corriols \& Doré, 1989; Gry, 1990).

El objetivo del presente trabajo fue, a través de la evaluación de poblaciones de espárrago, fijar criterios a partir de diferencias en rendimiento, calidad y efecto del sexo, para la selección de los mejores genitores que puedan ser utilizados en la construcción de materiales híbridos.

\section{MATERIAL Y MÉTODOS}

El ensayo se llevó a cabo en el campo experimental de la Facultad de Ciencias Agrarias de la Universidad Nacional de Rosario en la localidad de Zavalla $\left(33^{\circ} 1^{\prime} \mathrm{LS}, 60^{\circ} 53^{\prime} \mathrm{LO}\right)$, Provincia de Santa Fe, durante los años 1993 y 1994. Como materiales experimentales se utilizaron tres poblaciones del cultivar Argenteüil: Ohlsen-enke, Vilmorín y Clause $\left(\mathrm{P}_{1}, \mathrm{P}_{2}\right.$ y $\mathrm{P}_{3}$, respectivamente), dos cultivares de Precoz de Argenteüil $\left(\mathrm{P}_{4} \mathrm{y} \mathrm{P}_{6}\right)$, el híbrido todo macho AM771 $\left(\mathrm{P}_{5}\right)$ y la población tetraploide Violeta de Albenga $\left(\mathrm{P}_{7}\right)$, todos manejados como materiales blancos.
El diseño experimental fue de bloques completos aleatorizados con tres repeticiones, con 40 plantas de cada población por repetición. Las variables analizadas fueron: número de turiones (cantidad de brotes producidos por cada planta durante un período de 40 días a partir de la fecha de emergencia del primero), peso medio del turión en $\mathrm{g}$ ( $15 \mathrm{~cm}$ de longitud), diámetro medio del turión en $\mathrm{mm}$ (tomado en la base del tercio superior del turión), rendimiento en $\mathrm{g}$ (peso total de todos los turiones producidos), rendimiento de mercado en $\mathrm{g}$ (peso total de los turiones con diámetro $>12 \mathrm{~mm}$ ), días a cosecha (transcurridos desde la fecha de corte de la masa verde hasta la emergencia del primer turión), altura de la planta en $\mathrm{cm}$ (medida sobre el tallo más alto del helecho), peso de la masa verde en $\mathrm{g}$ (del helecho cortado a nivel del suelo al final del período vegetativo), número de tallos de la planta y perímetro de la planta en $\mathrm{cm}$ (tomado a nivel del suelo).

Todas las variables se midieron sobre planta individual. Las cinco primeras, fueron evaluadas en dos períodos de cosecha, mientras que las restantes se midieron durante los dos años de ensayo en las $\mathrm{P}_{1}, \mathrm{P}_{2}$ y $\mathrm{P}_{3}$, y sólo en 1994 en el resto de los materiales.

Las variables número de turiones, peso medio, rendimiento, rendimiento de mercado, masa verde y días a cosecha no presentaron una distribución normal, por lo cual se transformaron por: $\mathrm{X}^{0,5}+(\mathrm{X}+1)^{0,5}$ (Sokal \& Rohlf, 1967).

Con los datos obtenidos se realizó un ANOVA a dos criterios de evaluación, según el siguiente modelo:

$\gamma_{\mathrm{ijkl}}=\alpha_{\mathrm{i}}+\tau_{\mathrm{j}}+\beta_{\mathrm{k}}+\alpha \tau_{\mathrm{ij}}+\alpha \beta_{\mathrm{ik}}+\tau \beta_{\mathrm{jk}}+\alpha \tau \beta_{\mathrm{ijk}}+\varepsilon_{(\mathrm{ijk}) 1}$

donde:

$\gamma_{\mathrm{ijk} k \mathrm{l}}$ observación de la l-ésima planta en el k-ésimo sexo en la j-ésima población en el i-ésimo año;

$\alpha_{i \text { i: }}$ efecto del i-ésimo año;

$\tau_{\mathrm{j}}$ : efecto del $\mathrm{j}$-ésimo genotipo;

$\beta_{\mathrm{k}}$ : efecto del k-ésimo sexo;

$\alpha \tau_{\mathrm{ij}}$ : efecto de la interacción año-genotipo;

$\alpha \beta_{\text {ik }}$ : efecto de la interacción año-sexo;

$\tau \beta_{\mathrm{jk}}$ : efecto de la interacción genotipo-sexo;

$\alpha \tau \beta_{\mathrm{ijk}}$ : efecto de la interacción año-genotipo-sexo;

$\varepsilon_{(\mathrm{ijk}) 1}$ : error experimental

El análisis de agrupamiento de las poblaciones por sexo, se efectuó mediante el programa Fastclus, de SAS (1982), previo a un análisis de componentes principales.

\section{RESULTADOS Y DISCUSIÓN}

La variable más importante desde el punto de vista productivo es el rendimiento. El análisis de esta variable demostró diferencias significativas $(p<0,001)$ en el promedio de rendimiento en los dos años 
evaluados (Cuadro 1). El incremento fue del orden del 21,7\% (Cuadro 2), manifestándose a la vez diferencias en el comportamiento de las cultivares ensayadas, siendo estas diferencias provocadas por los bajos valores presentados por las poblaciones 6 y $7(24,10 \mathrm{~g})$ frente al resto de las poblaciones $(27,75 \mathrm{~g})$. Por otra parte, se demostró la existencia de una interacción genotipo-sexo significativa $(\mathrm{p}<0,05)$ ya que en la $\mathrm{P}_{6}$ las plantas estaminadas fueron superiores a las pistiladas en un $13 \%$, mientras que en el resto de las poblaciones no hubo diferencias entre sexos para esta variable, presentando un promedio por planta de $24,7 \mathrm{~g}$.

Paralelamente a lo demostrado por el rendimiento, el número de turiones manifestó un incremento del orden del $24 \%$ en el segundo año de evaluación y un comportamiento genotípico diferencial. En función del número de turiones pueden agruparse las poblaciones en tres categorías: I: poblaciones con un número promedio de turiones por planta superior a seis $\left(\mathrm{P}_{1}, \mathrm{P}_{2}\right.$ y $\left.\mathrm{P}_{3}\right)$; II: aquellas con un valor promedio entre cinco y seis turiones en promedio $\left(\mathrm{P}_{4}, \mathrm{P}_{5}\right.$ y $\left.\mathrm{P}_{6}\right)$ y III: poblaciones con un número promedio inferior a cinco turiones por planta $\left(\mathrm{P}_{7}\right)$. Para esta variable se presentó también un comportamiento diferencial de los sexos según el genotipo provocado por el hecho de que la $\mathrm{P}_{6}$ manifestó una más marcada superioridad de las plantas estaminadas frente a las pistiladas, mientras que en el resto de los materiales dichas diferencias son menores o nulas.

Por su parte, para peso medio del turión, se encontraron diferencias altamente significativas entre las poblaciones, entre los sexos y una marcada interacción genotipo-sexo. Los mayores valores para esta variable se presentan en las $\mathrm{P}_{7} \mathrm{y} \mathrm{P}_{4}(10,8 \mathrm{~g}$ de promedio) y los menores en la $\mathrm{P}_{1}(8,5 \mathrm{~g})$, siendo el promedio general de todos los materiales de $9,7 \mathrm{~g}$. Las plantas pistiladas presentan valores $8,8 \%$ superiores en todos los materiales, excepto en la $\mathrm{P}_{7}$, en donde no hay diferencias entre el peso medio de ambos sexos.

Cointry et al. (1996b) establecieron que el número de turiones, junto con el peso medio del turión, son las variables determinantes del rendimiento, concordando los datos del presente trabajo con dicha aseveración $(\mathrm{r}=0,81)$.

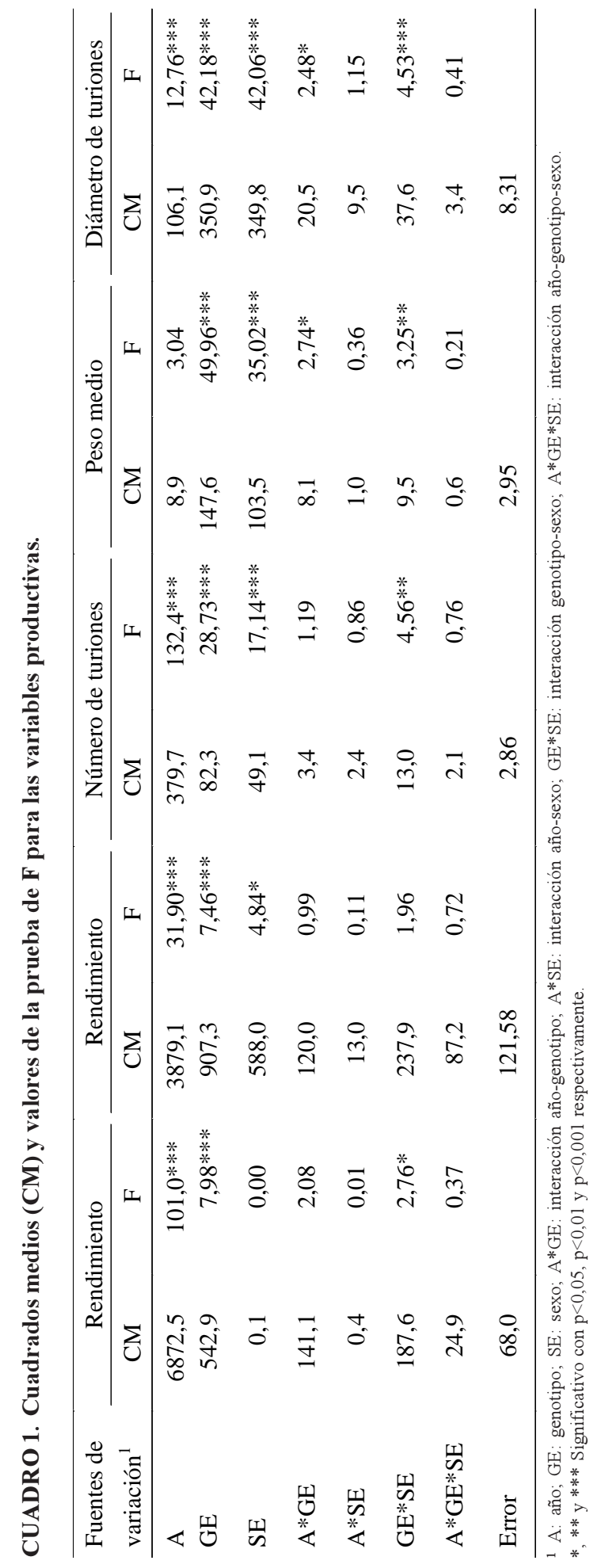

Pesq. agropec. bras., Brasília, v.35, n.6, p.1151-1157, jun. 2000 


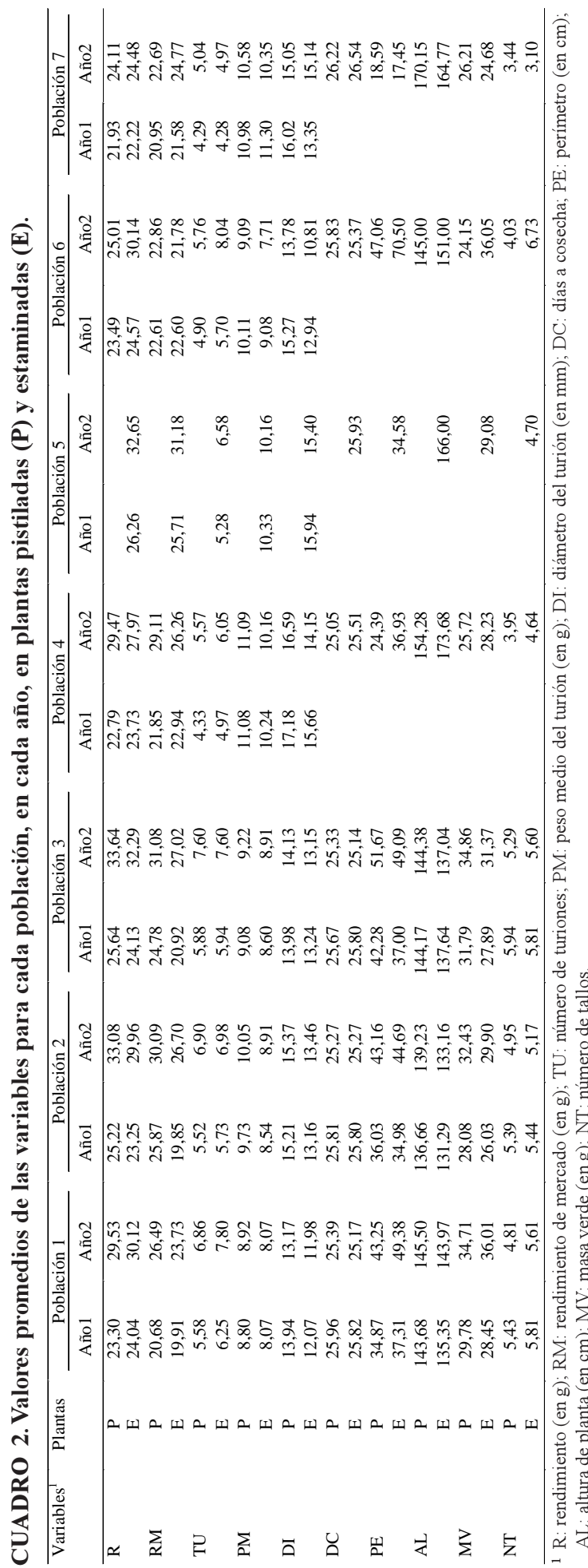

La variable diámetro de turión se comportó de la misma manera que el peso medio del turión, debido a la elevada correlación existente entre ellas $(\mathrm{r}=0,90)$. $\mathrm{P}_{4}, \mathrm{P}_{5} \mathrm{y}_{7} \mathrm{P}_{7}$ presentaron los mayores valores medios $(15,9 \mathrm{~mm})$, seguidas por el resto de las poblaciones con valores medios de 13,5 mm. Excepto para la $\mathrm{P}_{7}$, donde no hay diferencia entre los sexos, en el resto de las poblaciones ensayadas las plantas pistiladas presentaron valores superiores $(13,41 \%)$. En el segundo año de cosecha, el diámetro promedio se mantiene, excepto en $\mathrm{P}_{6} \mathrm{y}_{7}$, que muestran una disminución del $12 \%$, con lo cual podría explicarse la presencia de una interacción año-genotipo significativa.

Diversos autores (Ellison et al., 1960; Franken, 1970; Moon, 1976; Falloon \& Nikoloff, 1986) concuerdan con que las plantas estaminadas presentan mayor número de turiones mientras que las pistiladas tienen mayores diámetros; sin embargo, en los materiales aquí analizados, sólo la $\mathrm{P}_{6}$ demuestra esta superioridad en número de turiones y si bien las $\mathrm{P}_{1}$ a $\mathrm{P}_{6}$ presentan en cuanto a diámetro el mismo comportamiento señalado por estos autores, la $\mathrm{P}_{7}$ difiere de ellas.

Cointry et al. (1996a), trabajando con tres poblaciones de la cultivar de Argenteüil, encontró que el rendimiento aumenta con la edad de la esparraguera, a expensas del número de turiones, mientras que, tanto el diámetro como el peso medio de los mismos, se estabilizan en el segundo año de cosecha. En el presente trabajo puede concluirse que las $\mathrm{P}_{1}$ a $\mathrm{P}_{5}$ concuerdan con estas observaciones pero las $\mathrm{P}_{6} \mathrm{y}_{7} \mathrm{P}_{7}$ presentan un comportamiento diferente ya que si bien el número de turiones incrementa, el diámetro y el peso medio disminuyen ligeramente.

El rendimiento de mercado manifiesta diferencias entre poblaciones, entre años y entre sexos. Las $\mathrm{P}_{2}$, $\mathrm{P}_{3}$ y $\mathrm{P}_{5}$ presentan un rendimiento de mercado de 26,5 g en promedio por planta, $\mathrm{P}_{4} \mathrm{y}_{6}$, de 23,2 g, y $\mathrm{P}_{7}$ de 22,5 g. En las $\mathrm{P}_{2}$ y $\mathrm{P}_{3}$, las plantas pistiladas son superiores a las estaminadas en un $16 \%$ mientras que en el resto de las poblaciones no existen estas diferencias. Al igual que en rendimiento, esta variable presenta diferencias entre años, aumentando un $20 \%$ en el segundo período de cosecha provocado fundamentalmente por el incremento en el número de turiones. 
Para los días a brotación (Cuadro 3) sólo se encontraron diferencias significativas entre las poblaciones, siendo $\mathrm{P}_{7}$ la de producción más tardía (aunque cabe destacar que las diferencias no fueron mayores a 1,2 días), no existiendo, tampoco, diferencias entre sexos.

Para el peso de la masa verde, al igual que en el trabajo presentado por Legg et al. (1968), no se encontraron diferencias significativas entre sexos, manifestando valores superiores las $\mathrm{P}_{1}$ y $_{3}$ con $31,7 \mathrm{~g}$, intermedios las $\mathrm{P}_{2}$ y $\mathrm{P}_{5}$ con $29 \mathrm{~g}$ e inferiores las $\mathrm{P}_{4}, \mathrm{P}_{6}$ y $\mathrm{P}_{7}$ con $26,5 \mathrm{~g}$. Estas diferencias se vieron asociadas a variaciones en el número de tallos.

En cuanto a la interacción genotipo-sexo para masa verde, número de tallos y perímetro se debió fundamentalmente a las diferencias entre sexos de la $\mathrm{P}_{6}$.

Legg et al. (1968), Franken (1970) y Benson (1982) señalaron que las plantas pistiladas tienen mayor altura de planta. En el presente trabajo no se encontraron diferencias significativas entre sexos para esta última variable, pero se encontraron dife- rencias entre genotipos, siendo las $\mathrm{P}_{4}, \mathrm{P}_{5}$ y $\mathrm{P}_{7}$ superiores, con un promedio de $165,1 \mathrm{~cm}$, seguidas por $\mathrm{P}_{1}, \mathrm{P}_{3} \mathrm{y}_{6}$, con $143 \mathrm{~cm}$, por último, $\mathrm{P}_{2}$, con $134,8 \mathrm{~cm}$.

$\mathrm{El}$ análisis de componentes principales permitió establecer que los dos primeros componentes explicaron un $88 \%$ de la variación existente, siendo el primer componente el que explica la mayor variación (66\%). El primer componente (prin 1) es determinado, en las plantas estaminadas, por el número, el peso y el diámetro del turión y los días a cosecha y, el segundo componente (prin 2), por el rendimiento y el rendimiento de mercado. Para las plantas pistiladas, en cambio, el prin 1 es explicado por el número y el diámetro de los turiones y por el rendimiento, mientras que, el prin 2, por el diámetro del turión y el rendimiento de mercado.

En función de los componentes principales se conformaron, para las plantas estaminadas, cuatro grupos. El Grupo 1 (Cuadro 4), conformado por la $\mathrm{P}_{7}$, presenta bajos número de turiones, rendimiento $\mathrm{y}$ número de tallo, pero alto peso medio, diámetro $\mathrm{y}$

CUADRO 3. Cuadrados medios (CM) y valores de la prueba de $\mathrm{F}$ para las variables vegetativas.

\begin{tabular}{|c|c|c|c|c|c|c|c|c|c|c|}
\hline \multirow{2}{*}{$\begin{array}{c}\text { Fuentes } \\
\text { de variación }{ }^{1}\end{array}$} & \multicolumn{2}{|c|}{ Días a cosecha } & \multicolumn{2}{|c|}{ Perímetro } & \multicolumn{2}{|c|}{ Altura de planta } & \multicolumn{2}{|c|}{ Masa verde } & \multicolumn{2}{|c|}{ Número de allos } \\
\hline & $\mathrm{CM}$ & F & $\mathrm{CM}$ & F & $\mathrm{CM}$ & F & $\mathrm{CM}$ & F & $\mathrm{CM}$ & F \\
\hline $\mathrm{A}^{2}$ & 100,0 & $107,4 * * *$ & 31735,4 & $92,58 * * *$ & 1929,3 & 1,85 & 6840,1 & $45,78 * * *$ & 52,7 & $18,51 * * *$ \\
\hline GE & 4,9 & $5,29 * * *$ & 9763,0 & $28,48 * * *$ & 13945,2 & $13,36 * * *$ & 1197,2 & $8,01 * * *$ & 39,1 & $13,71 * * *$ \\
\hline SE & 0,0 & 0,08 & 2614,7 & $7,63 * *$ & 239,7 & 0,23 & 14,1 & 0,09 & 38,9 & $13,67 * * *$ \\
\hline $\mathrm{A}^{*} \mathrm{GE}$ & 0,4 & 0,43 & 161,5 & 0,47 & 807,6 & 0,77 & 271,7 & 1,82 & 0,1 & 0,06 \\
\hline $\mathrm{A} * \mathrm{SE}$ & 1,3 & 1,46 & 737,4 & 2,15 & 256,9 & 0,25 & 61,5 & 0,41 & 10,0 & 3,54 \\
\hline $\mathrm{GE}^{*} \mathrm{SE}$ & 1,4 & 1,58 & 1616,1 & $4,71 * * *$ & 1428,1 & 1,37 & 431,7 & $2,89 * *$ & 12,4 & $4,36^{* * * *}$ \\
\hline $\mathrm{A} * \mathrm{GE} * \mathrm{SE}$ & 0,7 & 0,77 & 11,6 & 0,03 & 575,3 & 0,55 & 80,8 & 0,54 & 0,6 & 0,22 \\
\hline Error & 0,93 & & 342,78 & & 1043,46 & & 149,41 & & 2,85 & \\
\hline
\end{tabular}

${ }^{1} \mathrm{~A}$ : año; GE: genotipo; $\mathrm{SE}$ : sexo; $\mathrm{A} * \mathrm{GE}$ : interacción año-genotipo; $\mathrm{A} * \mathrm{SE}$ : interacción año-sexo; GE*SE: interacción genotipo-sexo; $\mathrm{A} * \mathrm{GE} * \mathrm{SE}$ interacción año-genotipo-sexo.

2 El efecto de años, y sus interacciones, fue medido en las poblaciones 1,2 y 3

$*, * * \mathrm{y} * * *$ Significativo con $\mathrm{p}<0,05, \mathrm{p}<0,01$ y $\mathrm{p}<0,001$ respectivamente.

CUADRO 4. Valores de los componentes principales (Prin) para los diferentes grupos de poblaciones $\left(\mathbf{P}_{1}\right.$ a $\left.\mathbf{P}_{7}\right)$ para plantas estaminadas y pistiladas.

\begin{tabular}{|c|c|c|c|c|c|}
\hline \multirow[t]{2}{*}{ Prin } & \multirow[t]{2}{*}{ Valor Eigen } & \multicolumn{4}{|c|}{ Grupos en plantas estaminadas } \\
\hline & & $\mathrm{I}\left(\mathrm{P}_{7}\right)$ & II $\left(\mathrm{P}_{5}\right)$ & III $\left(\mathrm{P}_{2}\right.$ y $\left.\mathrm{P}_{4}\right)$ & $\mathrm{IV}\left(\mathrm{P}_{1}, \mathrm{P}_{3}\right.$ y $\left.\mathrm{P}_{6}\right)$ \\
\hline 1 & 7,03 & $-4,4$ & $-1,6$ & $-0,4$ & 2,3 \\
\hline 2 & 1,72 & $-1,5$ & 2,5 & $-0,1$ & $-0,2$ \\
\hline \multirow[t]{3}{*}{3} & 0,86 & 0 & $-0,3$ & $-0,05$ & 0,05 \\
\hline & & \multicolumn{4}{|c|}{ Grupos en plantas pistiladas } \\
\hline & & $\mathrm{I}\left(\mathrm{P}_{7}\right)$ & II $\left(\mathrm{P}_{4}\right)$ & III $\left(\mathrm{P}_{6}\right)$ & $\mathrm{IV}\left(\mathrm{P}_{1}, \mathrm{P}_{2}\right.$ y $\left.\mathrm{P}_{3}\right)$ \\
\hline 1 & 6,56 & $-3,7$ & $-1,9$ & $-0,8$ & 2,1 \\
\hline 2 & 2,26 & $-0,8$ & 2,3 & $-1,4$ & $-0,03$ \\
\hline 3 & 0,79 & 1,0 & $-0,3$ & $-1,5$ & 0,3 \\
\hline
\end{tabular}


días a cosecha. Si bien este grupo presenta características interesantes en cuanto a calidad de turión y producción tardía, para elegir genitores más apropiados, debería centrarse la atención en el Grupo 2 (población 5) que presenta los mayores rendimientos y rendimientos de mercado, con altos número de turiones, peso medio, diámetro y días a cosecha. El Grupo $3\left(\mathrm{P}_{2}\right.$ y $\left.\mathrm{P}_{4}\right)$, presenta un comportamiento intermedio entre el Grupo 2 y el 4 $\left(\mathrm{P}_{1}, \mathrm{P}_{3}\right.$ y $\left.\mathrm{P}_{6}\right)$, teniendo, este último, bajos diámetro y rendimiento de mercado, pero alto rendimiento.

En las plantas pistiladas, el Grupo 1 presenta idénticas características al Grupo 1 de las plantas estaminadas, incluyéndose en él la misma población. El Grupo 2 presentó los mayores diámetro y rendimiento de mercado y los menores días a cosecha $\left(\mathrm{P}_{4}\right)$, por lo cual, interesa para el aumento de productividad, pero no para el carácter producción tardía. El Grupo 3 presentó bajos rendimiento de mercado y diámetro y altos días a cosecha $\left(\mathrm{P}_{6}\right)$ mientras que el Grupo $4\left(\mathrm{P}_{1}, \mathrm{P}_{2}\right.$ y $\left.\mathrm{P}_{3}\right)$ presenta los mayores número de turiones, altos rendimiento y diámetro, pero bajo peso medio de turiones.

\section{CONCLUSIONES}

1. Para elegir genitores femeninos con caracteres tales como altos rendimientos se debe recurrir a las $\mathrm{P}_{1}, \mathrm{P}_{2}$ y $\mathrm{P}_{3}$, las cuales presentan también alto número de turiones, mientras que, para alto rendimiento de mercado, es indicada la $\mathrm{P}_{4}$.

2. Como genitores masculinos se indican las $\mathrm{P}_{5}$, que aportan los mejores rendimientos y rendimiento de mercado, y $\mathrm{P}_{7}$ con caracteres como altos peso medio, diámetro y días a cosecha, pero teniendo en cuenta que esta última población presenta bajos rendimientos y que, por tratarse de un material tetraploide, puede presentar problemas de inviabilidad en los cruzamientos.

\section{REFERENCIAS}

BANNEROT, H.; DERIEUX, M.; THEVENIN, L.; ARNOUX, J. Résultats d' un essai comparatif de populations d' asperge. Annales de l' Amélioration des Plantes, Paris, v.19, p.289-324, 1969.
BENSON, B.L. Sex influences of foliar trait morphologhy in asparagus. HortScience, Alexandría, v.17, n.4, p.625-627, 1982.

COINTRY, E.L.; LOPEZ ANIDO, F.S.; GATTI, I.; FIRPO, I.T.; GARCÍA, S.M. Criterios para la selección de plantas elite en Espárrago. Noticiero de Biología, Viña del Mar, v.4, n.3, p.144, 1996a.

COINTRY, E.L.; LOPEZ ANIDO, F.S.; GATTI, I.; GARCÍA, S.M.; FIRPO, I.T. Comparative study of morphological and productive characters in blanched asparagus populations. Asparagus Research Newsletter, Palmerston North, v.13, n. 1/2, p.30-34, 1996b.

CORRIOLS, L. Fast cultivar evaluation in asparagus trials. Asparagus Research Newsletter, Palmerston North, v.1, n.2, p.10, 1983.

CORRIOLS, L.; DORÉ, C. Use of rank indexing for comparative evaluation of all-male and other hybrid types in asparagus. American Society for Horticultural Science Journal, Alexandria, v.114, n.2, p.328-332, Mar. 1989.

ELLISON, J.H.; SCHEER, D.F.; WAGNER, J.J. Asparagus yield as related to plant vigor, earliness and sex. American Society for Horticultural Science Proceedings, Alexandria, v.75, p.411-415, 1960.

FALLON, P.G.; NIKOLOFF, A.S. Asparagus: value of individual plant yield and fern characteristics as selection criteria. New Zealand Journal of Experimental Agriculture, Lower Hutt, v. 14, p.417-420, 1986.

FRANKEN, A.A. Sex characteristics and inheritance of sex in asparagus (Asparagus officinalis $\mathrm{L}$.). Euphytica, Dordrecht, v.19, p.277-287, 1970.

GONZALES CASTAÑÓN, M.L. Evaluation of male and female asparagus plants. Interest in obtaining male dioecious hybrids. Acta Horticulturae, Leuven, v.271, p.83-89, 1990. 
GRY, L. L' asperge profite d' une sélection de pointe. Semences et Progrès, Paris, v.65, p.3-16, 1990.

LEGG, P.D.; SOUTHER, R.; TAKATORI, F.H. Estimates of heritability in Asparagus officinalis L. from replicated clonal materials. American Society for Horticultural Science Proceedings, Alexandria, v.92, p.410-417, 1968.

LEY, J.P.; MONGET, M.; THEVENIN, L. L' amélioration de 1' asperge (Asparagus officinalis L.): application conjointe de méthodes statistiques descriptives et inférentilles a l' utilisation raisonnée des différences de production entre plantes mâles et femelles. Annales de l' Amélioration des Plantes, Paris, v.26, n.4, p.675-716, 1976.

MOON, D.M. Yield potencial of Asparagus officinalis L. New Zealand Journal of Experimental Agriculture, Lower Hutt, v.4, p.435-438, 1976.

SAS INSTITUTE (Cary, NC). User's guide: Statistics. Cary, NC, 1982. 584p.

SOKAL, R.R.; ROHLF, F.S. Biometry. San Francisco : W. H. Freeman, 1967. 832p.

THEVENIN, L. Les problèmes d' amélioration chez Asparagus officinalis L. I: Biologie et Amélioration. Annales de l' Amélioration des Plantes, Paris, v.17, n.1, p.33-66, 1967. 